\title{
Three-Dimensional Measurement of the Femur Using Clinical Ultrasound: Developing a Basis for Image Guided Intramedullary Nail Fixation of the Femur
}

\author{
Dominic I. Young ${ }^{1}$, Sean M. Staniforth ${ }^{1}$, and Richard W. $\mathrm{Hu}^{3}$ \\ ${ }^{1}$ McCaig Centre for Joint Injuries and Arthritis Research, University of Calgary \\ 2500 University Drive N.W., Calgary, AB, Canada T2N 1N4 \\ diyoung@ucalgary.ca \\ ${ }^{3}$ Calgary FootHills Hospital, Department of Orthopaedic Surgery \\ 140329 Street N.W., Calgary, AB, Canada T2N 2T9
}

\begin{abstract}
Purpose: Quantify the precision and accuracy in coordinate measurements of anatomic landmarks of the femur using spatially tracked ultrasound (US) images. Establish the limits on coordinate measurement errors required for accurate determination of bone fragment alignment during intramedullary (IM) nail fixation of femoral shaft fractures. Relevance: A surgical guidance system based on a three-dimensional (3D) representation of femoral anatomy from US images would eliminate the hazard of radiation exposure and potentially increase the accuracy of IM nailing procedures. Summary: Fiducial spheres (dia. $6.3 \mathrm{~mm}$ ) were embedded in a plastic femur to mark anatomic landmarks. The femur was suspended in a water tank and could be rotated about its long axis. An US probe was mounted to a track above the femur. Images were collected at $5 \mathrm{~mm}$ increments along the anterior, posterior, lateral and medial aspects. After the US experiment, fiducial centroid locations $(x, y, z$-coordinates) were measured in a coordinate measuring machine (CCM). Reconstructed fiducial positions from US images were compared to the CMM data to assess precision and accuracy. A numerical model relating errors in landmark coordinate measurements to rigid body alignment was implemented. The mean precision (std-dev.) in fiducial coordinate measurements was $1.69 \mathrm{~mm}$. Mean and maximum errors in fiducial positions were $17.65 \mathrm{~mm}$ and $58.01 \mathrm{~mm}$, respectively. At the observed level of accuracy in coordinate measurements, the model predicted rigid body rotation errors of $3.4(\mathrm{SD}=2.4)^{\circ}$ and translation errors of $4.7(\mathrm{SD}=3.2) \mathrm{mm}$. A proof-of-concept has been demonstrated in the use of clinical US to obtain a quantitative description of femoral anatomy in a 3D framework. The model of error limits provided a basis for assessing the capability of a tracked US system in the context of a clinical criterion for rotational alignment (anteversion angle). Accuracy requirements for landmark coordinate measurements were at the limits of the capability of the current US tracking system.
\end{abstract}

\section{Introduction}

Closed IM nail fixation is the treatment of choice for adult femoral shaft fractures. Compared to an open technique, which is complicated by a large surgical incision, closed fracture fixation reduces the risk of infection at the fracture site by 
using a small incision at the proximal end of the femur to introduce the IM nail [1]. A $\mathrm{C}$-arm image intensifier (fluoroscope) is used to provide radiographic images that guide the placement of the IM nail and positioning of bone fragments during the procedure.

Alignment of the two bone fragments may be described by translations along and rotations about three axes corresponding to the anterior-posterior (A-P), lateral-medial (L-M) and proximal-distal (P-D) directions, respectively. The fractured ends of the bone must be aligned in the A-P and L-M directions for IM nail insertion. Proper rotational alignment about the P-D axis, called femoral anteversion, is particularly critical for a successful outcome. Femoral anteversion is characterised by the anteversion (AV) angle, commonly defined as the angle between a tangent plane to the posterior aspect of the femoral condyles and the longitudinal axis of the femoral neck [2]. Many patients tolerate some torsion, but efforts should be made to reduce and stabilize the femoral shaft fracture with an anteversion angle difference of less than $15^{\circ}$ compared to the contralateral leg [3].

Without the image intensifier, current methods and results of femur fixation would not be possible. However, there are two important concerns regarding this technique. First, there is a potential for prolonged exposure of the operating room personnel and the patient to dangerous levels of radiation [4], [5], [6], [7]. Due to the high frequency of femur fractures there is a risk of repetitive exposure of the surgeon to radiation. Second, mal-reduction problems and complications such as errors in length/rotation and improper fixation have been associated with the procedure [3]. Even with the aid of the fluoroscope, considerable skill is required to mentally reconstruct the three dimensional (3D) positions of the bone fragments and surgical tools, relying on non-continuous two dimensional (2D) X-ray images to confirm their positions.

Pre-operative computed tomography (CT) scans are the basis for many image guided surgical systems in use or under development [8], [9], [10]. Studies have described the use of virtual 3D bone surfaces constructed from CT images to aid surgeons in orthopaedic procedures [11]. Recently, intra-operative US has been studied for its ability to locate and register fiducial markers during interactive imageguided neurosurgery [12], [13]. The use of intra-operative US has been demonstrated in providing 2D images for monitoring the alignment of bone fragments in simple or minimally comminuted fractures that required unlocked nailing of the femur [14]. A surgical guidance system based on US technology would avoid the hazard of ionising radiation and offer the potential for real-time, intra-operative, imaging of the patient's anatomy.

The present study was undertaken as a first step in the development of a guided surgical system for IM nail fixation based on US imaging. The main purpose was to demonstrate the application of a clinical US system to the problem of obtaining a 3D description of the bony anatomy of the femur. The first objective was to quantify the precision and accuracy of landmark coordinate measurements made from tracked US images. The second objective was to establish limits on the coordinate measurement errors required to obtain the degree of accuracy necessary for determining the alignment between proximal and distal fragments of the femur during IM nailing procedures. 


\section{Methods}

\subsection{Preparation and Image Acquisition}

A plastic model femur (length $455 \mathrm{~mm}$ ) was fitted with 26 fiducial markers placed at anatomical landmarks at the proximal and distal ends and along the mid-shaft. Fiducial markers consisted of nylon spheres (diameter $6.3 \mathrm{~mm}$ ) that were embedded in small divots drilled into the surface of the specimen. Plastic rods were inserted at both ends of the specimen, allowing for rotation about its longitudinal axis when mounted in a Perspex frame specifically designed for this purpose (Fig. 1).

The specimen and support frame were placed inside a water tank so that the rotational axis of the specimen was parallel to the long sides of the tank and roughly $100 \mathrm{~mm}$ below the surface of the water. A clamp was placed across two aluminum rails fixed to the top of the tank. An US probe (SSD-900 Diagnostic Ultrasound System with $7.5 \mathrm{MHz}$ Probe, Aloka Corporation, Japan) was placed in the clamp so that it could slide along the length of the specimen parallel to the axis of rotation with the transducer head just below the surface of the water (Fig. 1).

The axial position of the US probe was measured (within $\pm 2.5 \mathrm{~mm}$ ) using a ruler fixed to one of the aluminum rails. The rotational position of the specimen could be set at $90^{\circ}$ intervals, allowing the presentation of each of its four surfaces (anterior, posterior, lateral and medial) to the probe. The probe was oriented perpendicularly to the rotational axis of the specimen with the US image-plane oriented to obtain axial cross-sections of the femoral surface in US images (Figs. 1, 2). Video output from the US system was connected to a capture board (LG-3 Scientific Frame Grabber, Scion Corporation, USA) controlled by Scion Image software (Scion Corporation, USA) running on a PC.

Sequential axial images of a single randomly assigned surface were captured as the US probe was translated along the rails at $5 \mathrm{~mm}$ increments. The specimen was then rotated to capture images of the remaining three surfaces in random order. Five trials each comprising four sets of surface images (anterior, posterior, lateral and medial) were completed. US images captured from the video display were stored in TIF format as 8-bit grayscale images.

\subsection{Analysis of US Images}

Each image from a particular series (e.g., anterior series of trial 1) was examined in sequence and compared with the physical specimen. The pixel coordinates of the centre of any visible fiducial marker were identified and then labelled according to the fiducial's location on the specimen. US images were also used to identify the location of the centre of rotation $\left(\mathbf{C}_{\mathbf{R}}\right)$, of the specimen based on the centres of the plastic rods inserted in the specimen.

\subsection{Reconstruction of Fiducial 3D Coordinates}

The second part of the analysis consisted of reconstructing the 3D locations of fiducials in a coordinate system based on the physical arrangement of the experimental set up (Fig. 1). The experimental coordinate system (ECS) was defined so that its origin was fixed at the centre of the proximal rotation support of the 
specimen. The $x, z$-plane was parallel to the short sides of the tank with the $y$-axis pointed distally and parallel to the specimen's axis of rotation. The $x-, y$ - and $z$-axes of the ECS corresponded roughly to the L-M, P-D and A-P axes of the model femur. The coordinate collection analysis produced the $x$ - and $z$-positions (horizontal and vertical, respectively) of fiducials in terms of image coordinates. The $x, z$-positions were scaled to millimetres (US image resolution $0.20 \mathrm{~mm}-$ pixel $^{-1}$ ). Because the specimen was rotated in $90^{\circ}$ intervals to image all four of its surfaces, the $x$ - and $z$ coordinates of fiducials identified in the posterior, lateral or medial views were reoriented in the ECS using a rotational transformation of $180^{\circ},-90^{\circ}$ or $90^{\circ}$ about $\mathbf{C}_{\mathbf{R}}$, respectively. Fiducial $x, z$-coordinates were combined with the $y$-coordinates (axial) to produce five reconstructed data sets $\left(x_{U S}, y_{U S}, z_{U S}\right)$ of 26 markers in terms of $x, y, z-$ coordinates in the ECS.

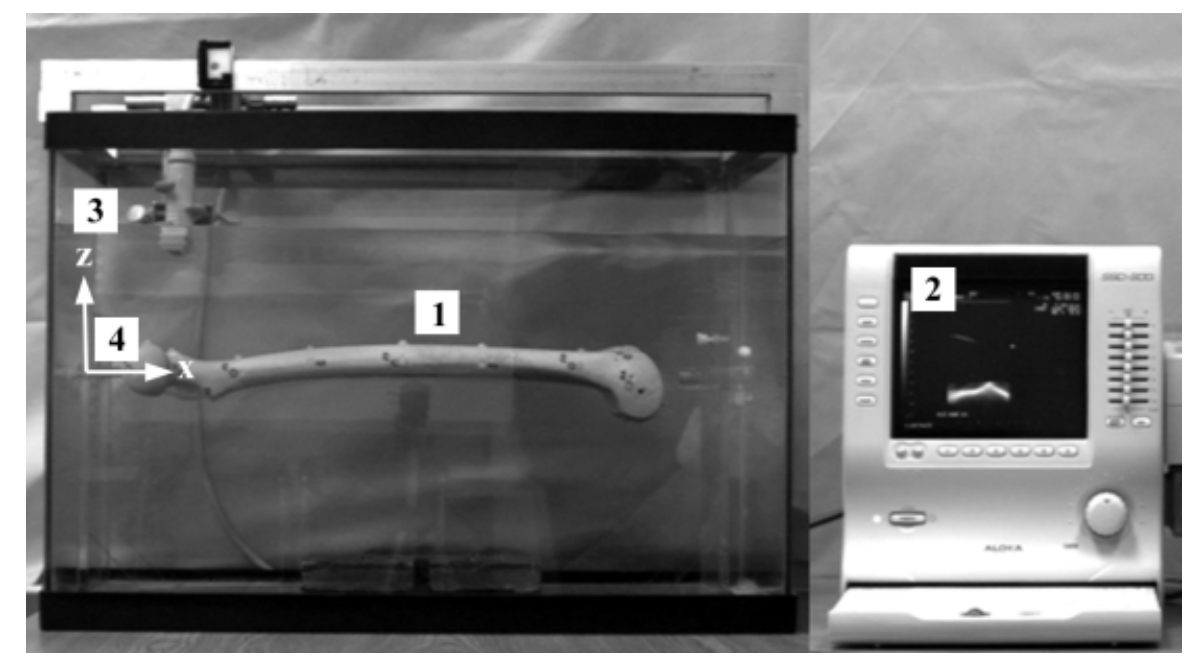

Figure 1. Experimental set-up. 1) Specimen (visible fiducials: lesser trochanter, medial epicondyle and femoral shaft markers). 2) Clinical US system (note the axial cross section displayed on the monitor). 3) US probe. 4) Experimental coordinate system (ECS)

\subsection{Precision and Accuracy}

Mean and standard deviation (SD), denoted by $\sigma$, for the five measurements of $x, y, z-$ coordinates were calculated for each fiducial. Precision, $\rho$, was quantified by the norm of the SDs:

$$
\rho=\sqrt{\sigma_{x}^{2}+\sigma_{y}^{2}+\sigma_{z}^{2}} .
$$

After the US experiment, the specimen and Perspex frame were fixed rigidly to the working surface of a coordinate measuring machine, or CMM (Mitutoyo MTI Corp., Japan, precision $\pm 0.050 \mathrm{~mm}$ ) and the 3D coordinates of the centroids of the 26 fiducial markers were measured. This calibrated data set $\left(x_{C M M}, y_{C M M}, z_{C M M}\right)$ was used to quantify the accuracy of the reconstructed data sets. The five sets of 26 reconstructed fiducial coordinates $\left(x_{U S}, y_{U S}, z_{U S}\right)$ were averaged and compared to the 
calibrated data set. Errors in $x-, y$ - and $z$-coordinates were calculated as $\left(x_{C M M}-x_{U S}\right)$, $\left(y_{C M M}-y_{U S}\right)$ and $\left(z_{C M M}-z_{U S}\right)$, respectively. Error in marker position, $\Delta$, was defined as the norm of the $x-, y$ - and $z$-errors:

$$
\Delta=\sqrt{\left(x_{C M M}-x_{U S}\right)^{2}+\left(y_{C M M}-y_{U S}\right)^{2}+\left(z_{C M M}-z_{U S}\right)^{2}} .
$$

\subsection{Modelling Accuracy Requirements}

To answer the second objective of the study, a simple model relating errors in landmark coordinate measurements to rigid body alignment (relative rotation and translation) was implemented. The model consisted of two 3D cubes (A and B) of volume $10^{6} \mathrm{~mm}$ with landmarks at their vertices. Body A was centred at the origin and aligned with the global coordinate system (GCS). Body B was rotated and translated with respect to $\mathrm{A}$. The rotation consisted of three equal Cardan angles and the translation of a 3D vector $\boldsymbol{d}=\left[\begin{array}{lll}100 & 100 & 100\end{array}\right] \mathrm{mm}$. Landmark $x, y, z$-coordinates on A and $\mathrm{B}$ were perturbed from their ideal positions by 100 sets of normally distributed random errors of mean magnitudes $0.5,5,15,25$ and $50 \mathrm{~mm}$. For five different Cardanic rotations $\left(5^{\circ}, 10^{\circ}, 15^{\circ}, 20^{\circ}\right.$ and $\left.25^{\circ}\right)$ of $\mathrm{B}$, the rigid body transformations between A and B (a $3 \times 3$ rotation matrix, M, and a $3 \mathrm{D}$ translation vector $\boldsymbol{t}$ ) were determined for the perturbed landmark locations [15]. Relative rotations (Cardan angles, $\alpha, \beta$ and $\gamma$ ) about and translations $\left(t_{x}, t_{y}, t_{z}\right)$ along the $x-y$ - and $z$-axes between $\mathrm{A}$ and $\mathrm{B}$ were resolved from the perturbed transformations and compared to the ideal rotations and translations.

The fiducial coordinate collection analysis, 3D reconstruction of fiducial coordinates and accuracy modelling were carried out in Matlab5.3 (The MathWorks Inc., USA) on a PC. Precision and accuracy analysis was carried out in Exce197 (Microsoft Corp., USA).

\section{Results}

\subsection{US Images of the Femoral Surface}

The "bone surface" of the specimen was clearly visible in all of the images. Large anatomic features (e.g., lesser trochanter, patellar notch and inter-condylar fossa) were easily identified. Finer details such as the $6 \mathrm{~mm}$ fiducial markers could also be resolved in the images. Occasionally, reflected sound waves reduced the sharpness of the surface boundary but this did not interfere with the reliable identification of fiducials (Fig. 2).

\subsection{Precision and Accuracy}

The femur's centre of rotation, $\mathbf{C}_{\mathbf{R}}$, was identified very reliably from the US images. There were usually from four to eight measurements of $\mathbf{C}_{\mathbf{R}}$ from each series of images. The largest SDs obtained in the horizontal and vertical components of $\mathbf{C}_{\mathbf{R}}$ were 1.1 and 1.5 pixels, respectively. Based on the image resolution, this variation was less than $0.5 \mathrm{~mm}$. Precision, $\rho$, in coordinate measurements from the five 
reconstructed data sets was found to be very high with mean and maximum values of $1.69 \mathrm{~mm}$ and $3.73 \mathrm{~mm}$, respectively. The mean error, $\Delta$, was $17.65 \mathrm{~mm}$ and the maximum error was $58.01 \mathrm{~mm}$. Errors in coordinate values were also examined as separate components: $\Delta_{\mathbf{x}}\left(x_{C M M}-x_{U S}\right), \Delta_{\mathbf{y}}\left(y_{C M M}-y_{U S}\right)$ and $\Delta_{\mathbf{z}}\left(z_{C M M}-z_{U S}\right)$. The mean values of $\Delta_{\mathbf{x}}, \Delta_{\mathbf{y}}$ and $\Delta_{\mathbf{z}}$ were $14.76,2.05$ and $9.49 \mathrm{~mm}$, respectively. The comparatively larger errors in the $x$ - and $z$-coordinates, corresponding to the horizontal and vertical directions in the US images, were largely responsible for the low accuracy of the fiducial location measurements.
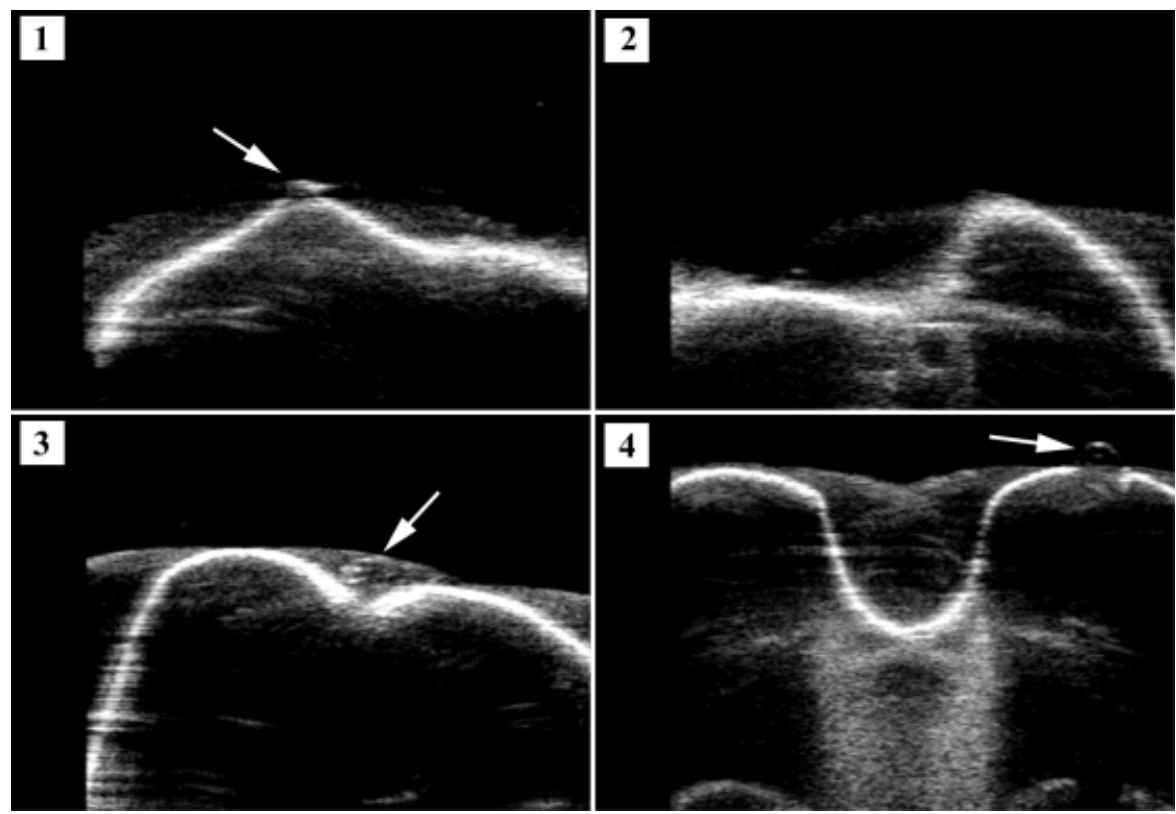

Figure 2. US images illustrating surface features of the proximal and distal ends of the femur. Fiducials are marked with arrows. 1) Anterior view of greater trochanter. 2) Posterior view of the greater trochanter and portion of the femoral neck. 3) Anterior view of the distal femur. 4) Posterior view of the femoral condyles

\subsection{Accuracy Requirements}

As expected, errors in rotation $(\alpha, \beta$ and $\gamma)$ and translation $\left(t_{x}, t_{y}, t_{z}\right)$ increased with the magnitude of landmark coordinate errors. Larger errors were observed for larger Cardanic rotations at each level of perturbation, however, alignment errors were not strongly dependent on the amount of rotation. The results are presented for values of $\beta$ and $t_{y}$, at a rotation of $15^{\circ}$ (Fig. 3). The largest mean error in fiducial coordinates obtained in the coordinate collection analysis was $\Delta_{\mathbf{x}}=14.76 \mathrm{~mm}$. At this level, the predicted rigid body alignment errors were $3.4(\mathrm{SD}=2.4)^{\circ}$ and $4.7(\mathrm{SD}=$ $3.2) \mathrm{mm}$ for $\beta$ and $t_{y}$, respectively. The largest amount of perturbation applied in the model $(50 \mathrm{~mm})$ corresponded to the maximum observed errors in fiducial coordinates. At this level, the predicted alignment errors were $11.2(\mathrm{SD}=8.8)^{\circ}$ and $15.5(\mathrm{SD}=$ $10.8) \mathrm{mm}$ for $\beta$ and $t_{y}$, respectively. 

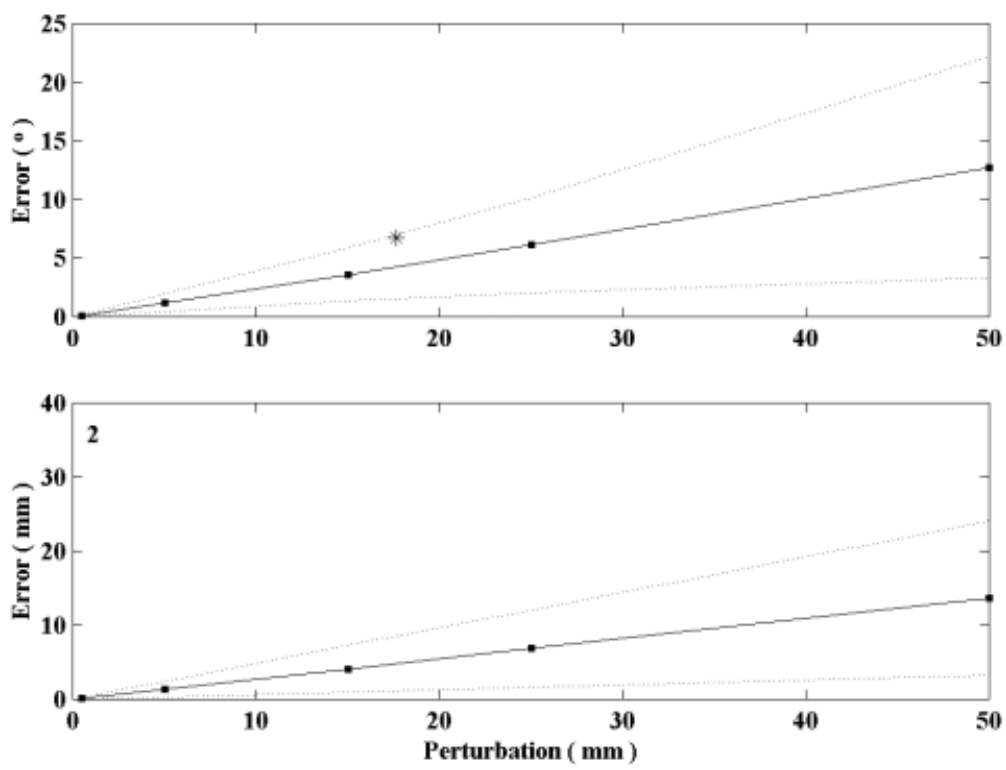

Figure 3. Accuracy model results for a nominal rotation $\beta=15^{\circ}$ and translation $t_{y}=100 \mathrm{~mm}$. Dashed lines indicate $S D$ in the error results ( $n=100$ perturbations). 1) Errors in rotation, $\beta$. Clinical accuracy criterion marked with an asterisk. 2) Errors in translation, $t_{y}$

\section{Discussion}

The main purpose of this investigation was to demonstrate the application of clinical US in measuring the relative positions of anatomic landmarks on the femur. Considering the simplicity of the "US tracking system", the precision $(\rho)$ in fiducial coordinate measurements was very high (within $\pm 2 \mathrm{~mm}$, on average).

A successful IM nail fixation procedure depends on the accurate assessement of the angular and linear position of the bone fragments. For example, a $15^{\circ}$ criterion for $\mathrm{AV}$ angle [3] indicates an error limit of $\pm 7.5^{\circ}$ for assessments of relative rotation between the proximal and distal fragments about the P-D axis. In order to achieve this accuracy, the errors in anatomic landmark coordinates $\left(\Delta_{\mathbf{X}}\right.$, etc.) must be within $\pm 18 \mathrm{~mm}$, according to the model of accuracy requirements (Fig. 3-1). The mean error in fiducial coordinate measurements $(\Delta=17.65 \mathrm{~mm})$ compares well to the accuracy requirements based on the $15^{\circ}$ criterion. However, it should be noted that alignment measurements depend on the accuracy in individual landmark coordinates. Therefore the range of fiducial coordinate errors of up to $58.01 \mathrm{~mm}$ were outside the limits of the $15^{\circ}$ criterion.

Fixing the orientation of the US transducer provided a means of "tracking" its position and reconstructing the $3 \mathrm{D}$ coordinates $\left(x_{U S}, y_{U S}, z_{U S}\right)$ of fiducials identified in US images. Coordinate errors were thought to be related to three assumptions that 
were made in order to execute the reconstruction: i) the US image plane was parallel to the $x, z$-plane of the ECS; ii) the centre of rotation of the specimen was parallel to the $y$-axis of the ECS; iii) the location of the centre of rotation, $\mathbf{C}_{\mathbf{R}}$, of points on the specimen's surface was identical for all images. The limitation of the third assumption was indicated by a "wobbling" observed at the proximal end of the specimen caused by misalignment of the rotation supports. The $x$ - and $z$-coordinates depended on a fiducial's location with respect to $\mathbf{C}_{\mathbf{R}}$. Larger errors in $x$ - and $z$-coordinates were likely caused by this motion. By comparison, errors in the $y$-coordinates were within the range of 2.0 to $5.0 \mathrm{~mm}$, which was consistent with the estimated accuracy in the axial position measurements $( \pm 2.5 \mathrm{~mm})$.

\section{Conclusion}

Results of the US imaging protocol demonstrated that fine details of femoral anatomy can be resolved and precisely located in a 3D framework. We have demonstrated an important proof-of-concept in the use of clinical US in providing a quantitative description of femoral anatomy. Our model of error limits provided a basis for assessing the capability of a tracked US system in the context of a clinical criterion for rotational assessment. The present system produced coordinate measurement errors that were within the limits indicated by the accuracy model. However, the range in coordinate errors needs to be reduced.

A plastic specimen in a water tank was an ideal model for producing sharp US images of the femoral surface. In a human limb, the acoustical properties of skin, fat and muscle as well as the presence of other bones at the hip and knee joints would likely affect the quality of US images and obscure details that were clearly visible in the experiment. A more physiologically representative model needs to be incorporated into future development of the system.

Currently, work is being done to incorporate optical tracking technology into the project. This will provide accurate spatial tracking of US images obtained using "free hand" scanning of the femur. In the long term, the goal is to develop a system that will give the surgeon an intuitive visual display of the alignment parameters relevant to IM nail fixation, a system that benefits from the safe and real time imaging capabilities of clinical US.

\section{References}

1. Mooney V., Claudi B.F.: Fractures of the shaft of the femur. In: Rockwood, C.A. and Green, D.P. (eds): Fractures in Adults. J.B. Lippincott Company, Philidelphia, (1984) 1396-1398

2. Lausten G.S., Jorgeson F., Boesen J: Measurement of anteversion of the femoral neck: ultrasound and computerised tomography compared. J. Bone Joint Surg. (Br). 75-B (1989) 799-803

3. Braten M., Terjesen T., Rossvoll I.: Torsional deformity after intramedullary nailing of femoral shaft fractures: Measurement of anteversion angles in 110 patients. J.Bone Joint Surg. (Br) 75(5) (1993) 7990-8003

4. Levin P.E., Schoen R.W., Browner B.D.: Radiation exposure to the surgeon during closed interlocking intramedullary nailing. J. Bone and Joint Surg. (Am) 69(5) (1987) 761-766 
5. Coetzee J.C., van der Merwe E.J.: Exposure of surgeons-in-training to radiation during intramedullary fixation of femoral shaft fractures. S. Afr. Med. J. 81(6) (1992) 312-314

6. Muller L.P., Suffner J., Wenda K., Mohr W., Rommens P.M.: Radiation exposure to the hands and the thyroid of the surgeon during intramedullary nailing. Injury. 29(6) (1998) 461-468

7. Smith G.L., Wakeman R., Briggs T.W.: Radiation exposure of orthopaedic trainees: quantifying the risk. J Royal College Surg. Edinburgh. 41(2) (1996) 132-134

8. Vannier M.W., Marsh J.L.: Three-dimensional imaging, surgical planning, and image-guided therapy. Radiol. Clin. North. Am. 34(3) (1996) 545-563

9. Glossop N., Hu R.: Effect of registration method on clinical accuracy of image guided pedicle screw surgery. Computer Assisted Radiology and Surgery, Proceedings of the 11th International Symposium and Exhibition, Berlin, Germany (1997) 884-888

10. Hu R., Glossop N., Steven D., Randle J.: Accuracy of image guided placement of iliosacral lag screws. 1st Joint CVRMed/MrCAS, Proceedings, Grenoble, France (1997) 593-596

11. Joskowicz L., Milgrom C., Simkin A., Tockus L., Yaniv Z.: FRACAS: a system for computer-aided image-guided long bone fracture surgery. Comput. Aided Surg. 3(6) (1998) 271-288

12. Hata N., Dohi T., Iseki H., Takakura K.: Development of a frameless and armless stereotactic neuronavigation system with ultrasonographic registration. Neurosurgery. 41(3) (1997) 608-613

13. Schreiner S., Galloway R.L., Lewis J.T., Bass W.A., Muratore D.M.: An ultrasonic approach to localization of fiducial markers for interactive, imageguided neurosurgery - Part II: Implementation and automation. IEEE Transactions on Biomedical Engineering. 45(5) (1998) 631-641

14. Mahaisavariya B., Laupattarakasem W.: Ultrasound or image intensifier for closed femoral nailing. J. Bone Joint Surg. (Br) 75(1) (1993) 66-68

15. Soederqvist I., Wedin P.A. Determining the movement of the skeleton using well-configured markers. J. Biomech. 26 (1993) 1473-1477 